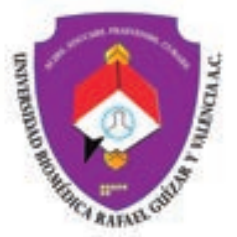

Vol. 8, Núm. 1

Enero-Junio 2021. pp. 19-27

doi: $10.35366 / 101201$

Información general actualizada

\title{
¿La rehabilitación mejora la función del cerebro dañado a través de la plasticidad cerebral y la regeneración neurológica? Parte 1
}

\author{
Does rehabilitation improve damaged brain function through \\ brain plasticity and neurological regeneration? 1 rth part
}

Francisco Aguilar Rebolledo*

\section{RESUMEN}

Introducción: La evidencia de la recuperación funcional después del daño cerebral se conoce empíricamente desde hace mucho tiempo. La plasticidad neuronal se refiere a los ajustes adaptativos del sistema nervioso central (SNC), y a la capacidad del cerebro para minimizar los efectos de las lesiones mediante la modificación de sus estructuras y función en su medio interno o externo. Objetivo: Conocer las bases neuronales de daño cerebral y la reorganización de sus funciones; tales como: desenmascaramiento, desarrollo de receptores sinápticos adicionales, factores tróficos, sinapsinas y regeneración de fibras nerviosas. Desarrollo: El proceso patológico puede afectar la corteza cerebral, los núcleos basales, el tallo cerebral, el cerebelo o la médula espinal. Existen estructuras funcionales en áreas específicas, si se afecta el lóbulo frontal izquierdo, la lesión producirá hemiparesia contralateral y trastorno del lenguaje. Gracias a la plasticidad cerebral se puede modificar y restaurar su propia estructura y funcionamiento. El siglo XXI ha sido declarado el «siglo del cerebro» por el enorme avance en las neurociencias, donde se promete introducir y desarrollar ideas importantes sobre el mecanismo del daño neuronal y al mismo tiempo cómo revertir

\section{ABSTRACT}

Introduction: The evidence for functional recovery after brain damage has been empirically known for a long time. Neural plasticity refers to the adaptive adjustments of the central nervous system (CNS), and the ability of the brain to minimize the effects of injuries by modifying its structures and function in its internal or external environment. Objetive: Know the neuronal bases of brain damage and the reorganization of its functions; such as: unmasking, development of additional synaptic receptors, trophic factors, synapsins, and nerve fiber regeneration. Development: The disease process can affect the cerebral cortex, basal ganglia, brainstem, cerebellum, or spinal cord. There are functional structures in specific areas, if the left frontal lobe is affected, the lesion will produce contralateral hemiparesis and language disorder. Thanks to brain plasticity, its own structure and functioning can be modified and restored. The 21st century has been declared the "century of the brain» due to the enormous advance in neurosciences, where it promises to introduce and develop important ideas about the mechanism of neuronal damage and at the same time how to reverse

* Rector de la Universidad Biomédica Rafael Guízar y Valencia.

Correspondencia:

Académico M. en C. Dr. Francisco Aguilar Rebolledo

Ángel Garrido No. 2, Colonia Represa del Carmen C.P. 91050, Xalapa, Veracruz, México.

E-mail: fran_aguilar_invest@yahoo.com.mx

Recibido: 30-03-2021. Aceptado: 20-06-2021.

Citar como: Aguilar RF. ¿La rehabilitación mejora la función del cerebro dañado a través de la plasticidad cerebral y la regeneración neurológica? Parte 1. Plast Restaur Neurol. 2021;8 (1): 19-27. https://dx.doi.org/10.35366/101201 
el proceso de la muerte celular. El desarrollo de estudios como la resonancia magnética funcional (RMF), la tomografía por emisión de positrones (PET) y la magnetoencefalografía (MEG) nos permiten conocer la función in vivo. Conclusión: La expresión de la plasticidad cerebral y la capacidad de las neuronas para cambiar su función, perfil químico o estructura son el nuevo marco conceptual para la contribución en la restauración del cerebro.

Palabras clave: Plasticidad cerebral, restauración neurológica, recuperación funcional. the process of cell death. The development of studies such as functional magnetic resonance, positron emission tomography and magnetoencephalography allow us to know the function in vivo. Conclusion: The expression of brain plasticity and the ability of neurons to change their function, chemical profile or structure are the new conceptual framework for the contribution in the restoration of the brain.

Keywords: Brain plasticity, neurological restoration, functional recovery.

\section{INTRODUCCIÓN}

La plasticidad cerebral es la adaptación funcional del Sistema Nervioso Central (SNC) para minimizar los efectos de alteraciones estructurales o fisiológicas, cualquiera que sea la causa y que puedan ocurrir en cualquier momento de la vida. ${ }^{1}$ Esto es posible gracias a la capacidad de cambio estructural-funcional que tiene el sistema nervioso (SN) debido a la influencia de factores tanto endógenos como exógenos; en otras palabras, esto expresa la asombrosa capacidad adaptativa del SNC para minimizar los efectos de lesiones, a través de la modificación de su propia organización estructural y funcional.

El grado de recuperación guarda una relación directa con algunos factores que intervienen, como son la edad, localización del área dañada, cantidad de tejido dañado, así como los programas de rehabilitación a los que es sometido el paciente, intervención oportuna, factores ambientales y psicosociales. ${ }^{2}$ Cabe mencionar que aun en una lesión parcial en el cerebro inmaduro, la recuperación es mayor que en un cerebro adulto, pero se reconoce que en todas las edades hay probabilidades exitosas de recuperación. ${ }^{3}$

Esto se logra a través de vías motoras y sensitivas que intervienen en la plasticidad, por medio de mecanismos a nivel histológico, bioquímico y fisiológico.

La voluntad del paciente por recuperarse y el buen criterio y conocimiento del neurólogo y del rehabilitador pueden conseguir resultados admirables en la recuperación de lesiones cerebrales que no sean masivas y que no sean de carácter degenerativo. ${ }^{4}$

Prácticamente todas las funciones cerebrales pueden verse beneficiadas de una lesión debido a la recuperación de la función neurológica, por ejemplo, el lenguaje, la escritura, la capacidad cognoscitiva, la conducta y las emociones.

En los traumatismos craneoencefálicos se provocan tanto lesiones directas como indirectas, destrucción traumática y destrucción bioquímica. La administración de sustancias que bloquean neurotransmisores excitadores o por aplicación de anticuerpos contra sustancias inhibidoras del crecimiento neurítico que ocurren tras el traumatismo, permite la recuperación de una porción de los axones afectados de la zona lesionada en el SNC. ${ }^{5}$

A continuación, se presenta una serie de líneas de investigación actuales y futuras para el incremento de las bases científicas de la rehabilitación neurológica:

1. Regeneración del SNC.

2. Potencial biológico de la función del tejido cerebral.

3. Mecanismos adaptativos que siguen a la lesión del $\mathrm{SN}$.

4. Capacidad del SNC para restaurar su función.

5. Transferencia de la función nerviosa y ciencia neural computacional.

6. Psicología cognoscitiva de las funciones nerviosas.

7. Neurofarmacología de la recuperación. ${ }^{6}$

\section{PERIODOS CRÍTICOS DE LAS FUNCIONES CEREBRALES}

Los periodos críticos tienen especial importancia para la vida en etapas tempranas, tienen relación directa con factores ambientales que funcionan facilitando y estimulando su aparición y desarrollo; durante las etapas temporales de adquisición de dichas habilidades se les denomina periodos críticos -el periodo en el cual la experiencia y la actividad nerviosa que lo reflejan tienen un efecto máximo sobre la adquisición o la ejecución hábil de una conducta particular-..$^{6-8}$

Los periodos críticos para las habilidades sensitivas y los comportamientos complejos son más prolongados y terminan de forma mucho menos brusca. En algunos casos como la adquisición del lenguaje en los seres humanos, las influencias instructivas detalladas del medioambiente (es decir, la exposición al lenguaje y las oportunidades de imitación y repetición, como ocurre en el retraso primario del lenguaje, en el cual el niño llega a los dos años diciendo sólo unas cuantas palabras y frecuentemente se confunde con autismo, cuando en realidad lo que requiere el niño es precisamente 
influencias instructivas del medioambiente, es decir, que el niño esté en contacto con otros niños, lo que sucede en la guardería y el kínder, son el mejor estímulo para desarrollar el lenguaje). Estos estímulos son necesarios durante un periodo intenso para asegurar el desarrollo normal del comportamiento. La disponibilidad de experiencias instructivas desde el medioambiente y la capacidad nerviosa para responder a ellas son la clave para completar con éxito el periodo crítico. ${ }^{9}$

Un periodo crítico se define como el tiempo durante el cual un comportamiento dado es especialmente susceptible a las influencias ambientales específicas y en realidad requiere de ellas para desarrollarse con normalidad. Estas influencias pueden ser tan sutiles como los estímulos progresivos de la luz o el sonido que encuentra un lactante o la instrucción precisamente articulada en el preciso leguaje nativo (o uno extranjero) para alcanzar y lograr una palabra fluente y una comprensión precisa. Una vez que termina este periodo, las características centrales de este comportamiento en su mayor parte no se ven afectadas por la experiencia posterior. Por el contrario, es difícil o a veces imposible remediar la falta de exposición a estímulos apropiados durante el periodo crítico -los estudios del Dr. Aguilar y colaboradores en niños privados de estímulos en el periodo crítico del lenguaje constituye un ejemplo de que la falta de estímulos una vez que ha terminado el periodo crítico les dificulta el desarrollo del lenguaje de una manera precisa, tanto el fluente como el de la comprensión-. ${ }^{10}$ En la mayoría de los mamíferos, incluidos los seres humanos, los periodos críticos parecen basarse particularmente en los cambios en la organización y la función de los circuitos de la corteza cerebral. Por lo tanto, gran parte de nuestra explicación sobre los periodos críticos deberá enfocarse en las consecuencias de la experiencia y la actividad para influir en el crecimiento, las conexiones y la función de la corteza cerebral desde el nacimiento hasta el final del periodo de desarrollo del adolescente.

\section{Correlaciones celulares y moleculares de la plasticidad durante los periodos críticos}

Sin duda, los patrones de actividad para modificar las conexiones nerviosas son iniciados por señales generadas por actividad sináptica asociada a la experiencia sensitiva, el procesamiento perceptual y cognitivo o el rendimiento motor. Los neurotransmisores y algunas moléculas de señalización, incluidos los factores neurotróficos son los candidatos obvios para iniciar los cambios que ocurren en las actividades correlacionadas o repetidas impulsadas por la experiencia. Los neurotransmisores excitadores como el glutamato y los receptores como el NMDA son clave para comprender la competencia dependiente de actividad y la modificación de los circuitos durante el desarrollo y la plasticidad sináptica en el adulto.

La experiencia de la corteza visual es un ejemplo de la plasticidad de las columnas que se desarrollan para la función de la visión. Es destacada la acción del calcio $(\mathrm{Ca}+)$ y de otras moléculas secretadas como las neurotrofinas, las cuales conducen a modificaciones del citoesqueleto, dependientes de la fosforilación y a cambios en la ramificación dendrítica y axónica, las interneuronas gabaérgicas son particularmente sensibles a una señalización del factor de desarrollo a la transcripción de genes de neurotrofinas como BDFN.

Un factor extremadamente importante en los cambios tardíos es el patrón de estimulación de la vía visual. Es bien conocido el experimento de Hubel y Wiesel en relación a la etapa crítica del desarrollo de la visión, estos investigadores demostraron que si el párpado del ojo de un gatito es suturado durante los meses críticos del desarrollo visual normal, el ojo quedará permanentemente ciego después de haber sido retiradas las suturas (Figura 1).

Los histogramas representan la cantidad de células que caen en una de las siete categorías de dominancia ocular, definidas sobre la base de la frecuencia de la actividad de los potenciales de acción producidos en las neuronas en la corteza cerebral visual primaria de los gatos adultos normales, en los tres casos, las lecturas experimentales se realizaron en gatos de 38 meses de vida, momento en el cual se enfocaron las luces sobre los dos ojos abiertos para inducir respuestas sobre la corteza visual (Figura 1) distribución normal a partir de un gran número de neuronas en la corteza visual primaria. Las células del grupo 1 fueron activadas exclusivamente por el ojo contralateral; las células del grupo 7 por el ojo homolateral (Figura 1), se cerró un ojo de un gato recién nacido desde una semana después del nacimiento hasta los 2.5 meses de vida. Después de este tiempo se abrió el ojo y el gato maduró normalmente hasta los 38 meses. Obsérvese que la privación fue relativamente breve -el ojo suturado había estado abierto durante los 35.5 meses de vida del gato-, incluso en este caso la luz presentaba al ojo abierto, pero transitoriamente cerrado no produjo respuestas eléctricas en las neuronas corticales visuales. Las únicas células con respuesta visual respondieron al ojo contralateral (no cerrado) (Figura 1); un periodo mucho más prolongado de oclusión monocular en un gato adulto tiene poco efecto sobre la dominancia ocular (aunque está disminuida la actividad cortical global). La mayoría de las células que responden son activadas por ambos ojos. Además, algunas células del 
(A) Adulto normal

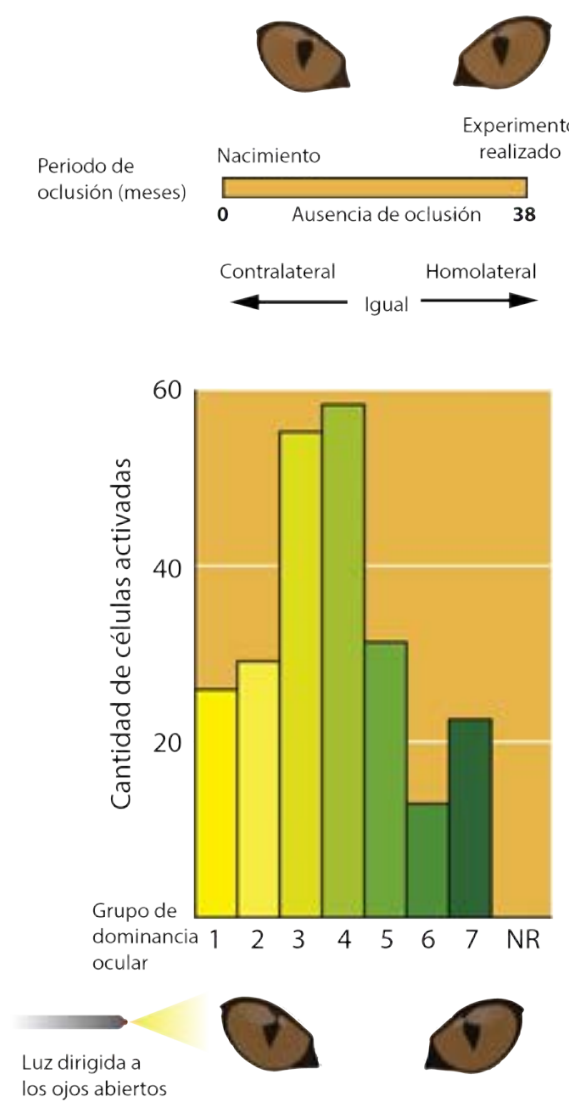

(B) Privación monocular en gatitos
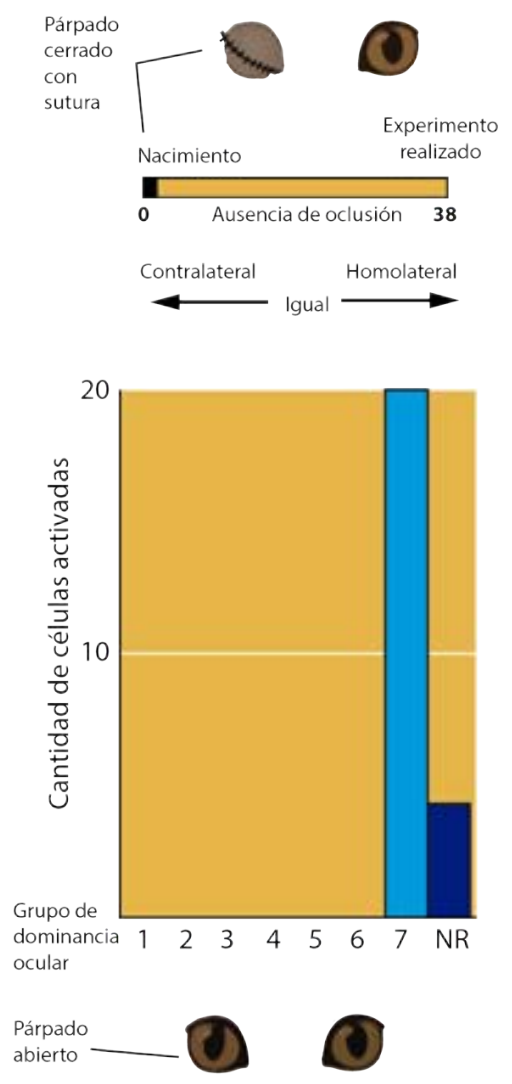

(C) Privación monocular en el adulto
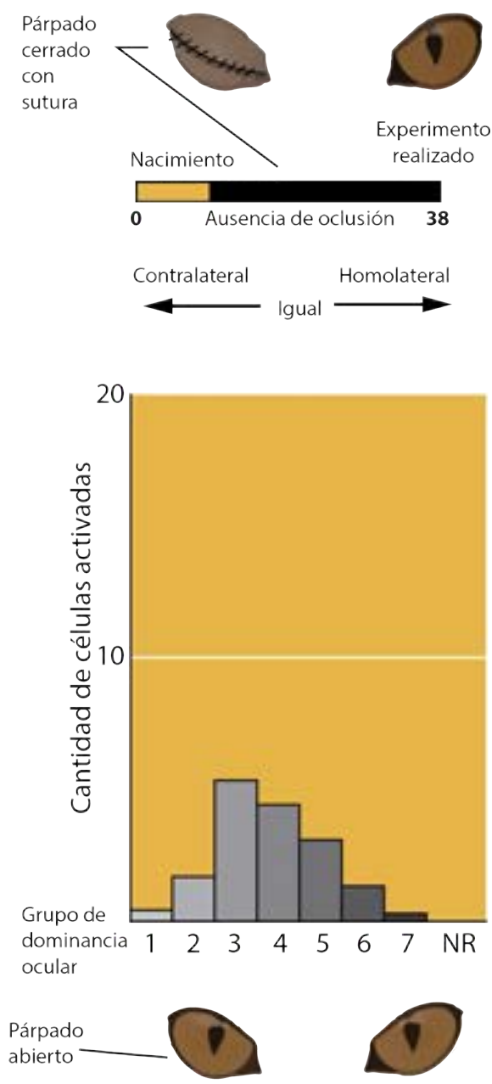

Figura 1: Periodos críticos de la visión. Experimento en gatos por Hubel y Wiesel (premio Nobel 1981). En el cual cerraron el ojo de gato recién nacido y lo destaparon después de cierto tiempo observando la pérdida de la visión por falta de desarrollo columnar y visual en la corteza occipital. Efecto del cierre temprano de un ojo sobre la distribución columnar de las neuronas corticales activadas por la estimulación de ambos ojos.

Con Permiso de editorial Interamericana.

grupo 1 y 2 responden única o principalmente al ojo cerrado (Figura 2).

El gato puede vivir aún por cinco años o más con sus párpados funcionando normalmente, pero ciego. Este extraordinario hallazgo a través de un experimento bien organizado demostró el periodo crítico para el desarrollo de la visión. Sin embargo, Chow y Steward hicieron la pregunta crítica: ¿puede obtenerse recuperación de la visión con un programa de entrenamiento (rehabilitación)?

Ellos fueron capaces de demostrar que no sólo es posible obtener alguna función (visión), sino que también se registraron:

1. Constantemente cambios fisiológicos (incremento en el número de células binoculares en la corteza visual).
2. Morfológicos (cambios en el cuerpo geniculado lateral).

Estos investigadores también anotaron que el uso del sistema tradicional de gratificación fue insuficiente, los gatos requerían periodos de complacencia y de mayor atención (para ello se establecieron lazos afectivos con los experimentadores); además de los métodos utilizados se procuró desarrollar un plan de demanda, es decir, una rehabilitación intensiva, pero sin llegar a la frustración, y los resultados siempre fueron mayores a los esperados. ${ }^{11,12}$

En otro modelo animal con ratas Rosenzweig, se ha demostrado en modelos apropiados que ambientes enriquecidos, consistentes, en jaulas con cierto número de «juguetes» y otros estímulos placenteros, inducen cambios: morfológicos, fisiológicos, neuroquímicos y conductuales. 
Los últimos estudios experimentales han intentado correlacionar la actividad de estos sistemas con el periodo crítico de plasticidad neuronal y la facilitación de cambios estructurales en la corteza entre las diferentes funciones de otros lóbulos cerebrales (Figura 3). Investigaciones sobre los cambios en el sistema visual analizan estos aspectos en la corteza visual y los extrapolan ante las evidencias clínicas al resto de la corteza cerebral. Estos estudios se basan en modelos animales, pero pueden servirnos para establecer hipótesis de recuperación funcional en humanos.

\section{Audición. Periodos críticos}

Los implantes sensoriales fueron de los primeros dispositivos sensoriales en cosechar éxitos. Su función es sustituir a los órganos de los sentidos, recogiendo los estímulos del entorno y enviando señales al sistema nervioso para que sean procesados como lo haría de manera natural el cerebro. Un primer nivel de la tecnología de implantes sensoriales ya muy extendida (afortunadamente) es el implante coclear, un aparato que recoge los sonidos del exterior y los convierte en impulsos nerviosos en el nervio auditivo, restaurando así la capacidad auditiva en personas sordas.

\section{¿Qué finalizan los periodos críticos?}

Al respecto, todavía no hay una respuesta absoluta del porqué finalizan estos periodos críticos; no obstante, a continuación se presentan tres posibles hipótesis:

1. La plasticidad disminuye cuando cesa el crecimiento axónico.

2. La plasticidad disminuye cuando la transmisión sináptica madura.

3. La plasticidad disminuye cuando se limita a la activación cortical. ${ }^{10}$

\section{(A) Adulto normal}
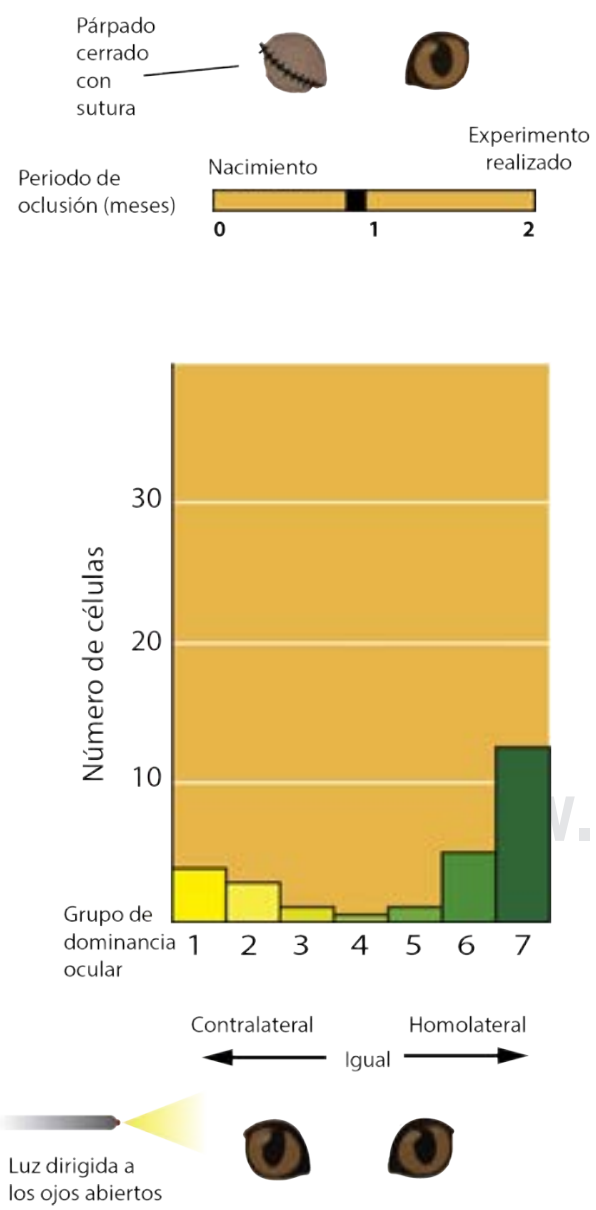

(B) Privación monocular en gatitos
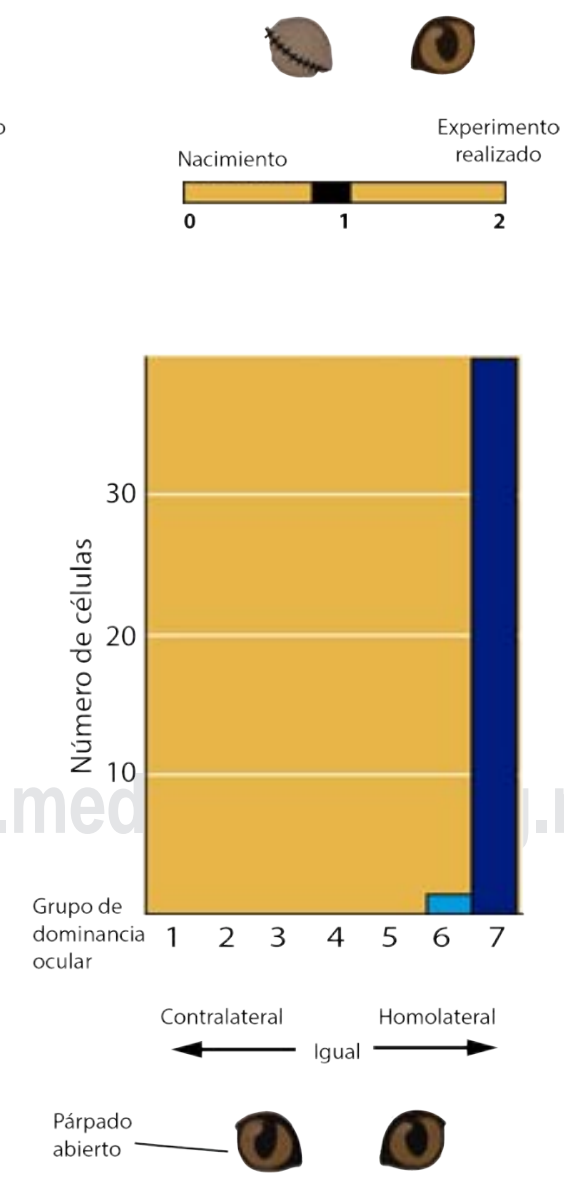

Figura 2:

Consecuencias de un periodo breve de oclusión monocular en la cima del periodo crítico en el gato. A) En este ejemplo, tan sólo tres días de oclusión produjeron un desplazamiento importante de la activación cortical a favor del ojo que permaneció abierto. B) Seis días de oclusión produjeron un desplazamiento de la activación cortical a favor del ojo no ocluido que es casi completo, como el obtenido por los 2.5 meses de oclusión. Con Permiso de editorial Interamericana. 
Tabla 1: Mecanismos de plasticidad en el sistema nervioso en desarrollo.

\begin{tabular}{ll}
\hline Periodo & Mecanismo \\
\hline Desarrollo temprano (relativamente sujeto a un programa genético) & Sobreproducción de neuronas \\
& Desarrollo exuberante de axones \\
& Retoños dendríticos exuberantes \\
Desarrollo tardío (modificable por el ambiente) & Sobreproducción de sinapsis \\
& Muerte neuronal programada \\
Factores que modifican el desarrollo tardío & Interrupción axonal \\
& Proliferación de dendritas \\
& Eliminación de sinapsis \\
& Cambios en el tamaño del objetivo \\
& Actividad neuronal \\
& Factores de desarrollo neuronal \\
& Cambios endocrinos \\
& Cambios metabólicos \\
\hline
\end{tabular}

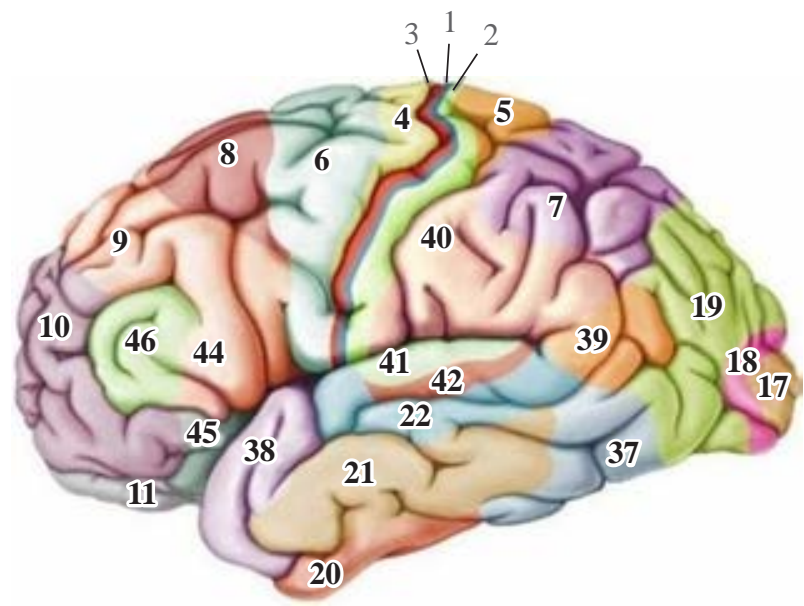

Figura 3: Integración de distintas funciones cerebrales, equipotenciales entre diferentes lóbulos cerebrales.

\section{MECANISMOS DE RESTAURACIÓN NEUROLÓGICA DESPUÉS DE DAÑO CEREBRAL}

Las investigaciones actuales dan como resultado esa extraordinaria flexibilidad, a causa de la cual el cerebro sufre constantes cambios tanto químicos como físicos en respuesta a su entorno; dichos cambios son lo que ahora se conoce como plasticidad. En determinadas lesiones cerebrales en las que se ha perdido el funcionamiento de un área, se tiene la posibilidad de recobrar parcial o totalmente dicha función a través de la restauración con una intervención y/o estimulación oportuna, es decir, a través de un trabajo continuo y multidisciplinario.

Factores que influyen en la restauración nerviosa: ${ }^{13}$
1. Edad.

2. La naturaleza y la evolución de las lesiones.

3. La velocidad de la producción del daño.

4. El tiempo entre el traumatismo y la atención del paciente.

5. La calidad de atención.

6. Las sustancias farmacológicas.

7. La cantidad de tejido viable.

8. El número de estructuras lesionadas.

9. Los sistemas de neurotransmisión involucrados.

10. Las etapas del desarrollo en las que se encuentra el paciente.

11. El aprendizaje y estimulación del medioambiente.

Otro elemento importante a considerar es el referente a los mecanismos de cómo ocurren los cambios en el sistema nervioso después de una lesión (Figura 4).

\section{PLASTICIDAD CEREBRAL Y REORGANIZACIÓN DEL SISTEMA NERVIOSO LESIONADO}

Después de la lesión en el SNC existen mecanismos naturales de naturaleza plástica que realizan reorganización nerviosa. En la lesión motora cerebral muchas fibras descendentes se interrumpen, algunas se degeneran y requieren estímulos para detonar un extenso mecanismo de reorganización cerebral a través de formación de retoños axonales a varios niveles desde la corteza cerebral hasta la médula espinal; a nivel interneuronal, los ramos terminales extienden sus conexiones a otras interneuronas somáticas y de éstas interneuronas a la membrana de las motoneuronas que ocupan los espacios que dejan las fibras afectadas.

El resultado fisiológico inicial es la emergencia de respuestas exageradas de los reflejos a los impulsos 
periféricos, tales como los estímulos cutáneos, el estiramiento muscular, etcétera.

Por otro lado, aparece la liberación de los impulsos espinales con espasticidad y acortamiento muscular. La reorganización espinal junto con las estrategias de los centros superiores a través de vías descendentes permanecen intactas o parcialmente activas, tales como: haces reticuloespinales, rubroespinal y vestibuloespinal, los cuales extienden sus retoños axonales hasta nivel de las motoneuronas y envían impulsos de activación cortical motora ipsilateral.

Ambos mecanismos generan patrones anormales, tanto supra- como infraespinal, los cuales favorecen aún más la sobreactividad espinal. ${ }^{14}$

Al corregir con la toxina botulínica, el estiramiento muscular, la disminución de la contracción y la velocidad dependiente del incremento del reflejo de estiramiento a veces es suficiente para obtener una mejoría de la espasticidad y, en consecuencia, la mejoría del apoyo plantar neutro y la marcha. A nivel de centros superiores se elaboran estrategias con engramas motores para favorecer el movimiento normal con reorganización plástica a nivel espinal (Figura 5 A y B). ${ }^{13-15}$

\section{CONCLUSIONES}

La plasticidad neuronal es la capacidad de las áreas cerebrales o de grupos neuronales de responder funcional y neurológicamente supliendo las deficiencias funcionales correspondientes a la lesión.

La capacidad de las neuronas de asumir el papel de otra que esté lesionada se conoce como reorganización cerebral, y puede ser anatómica, fisiológica o estructural. Existe además la plasticidad sináptica y la posibilidad de crecimiento de nuevas sinapsis a partir de estímulos generados por liberación de factores de desarrollo, factores tróficos o modificación de neurotransmisores o de genes que estimulan nuevas redes neuronales.

Por lo tanto, el término plasticidad cerebral expresa la capacidad adaptativa del sistema nervioso para minimizar los efectos de las lesiones a través de modificar su propia organización estructural y funcional.

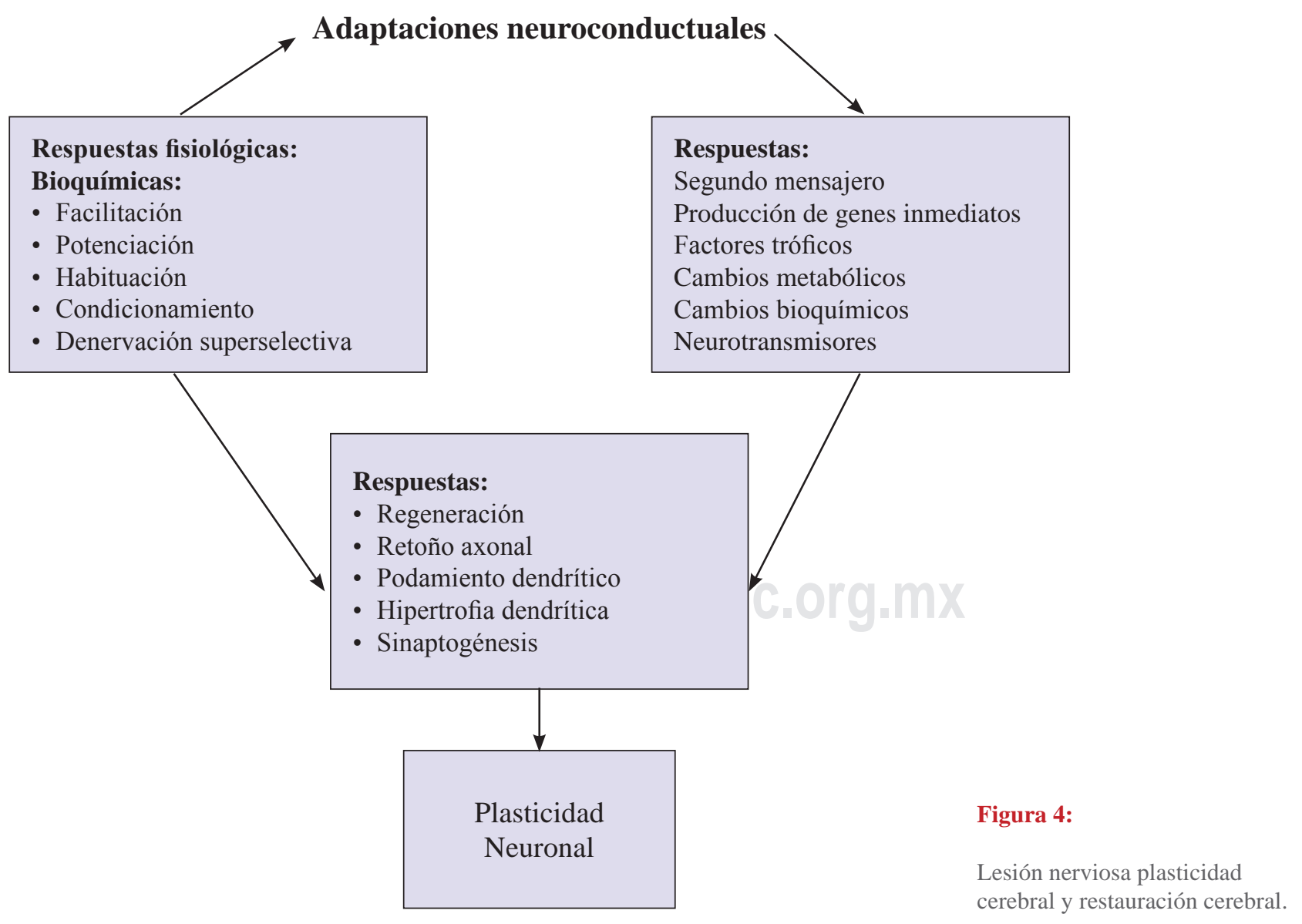



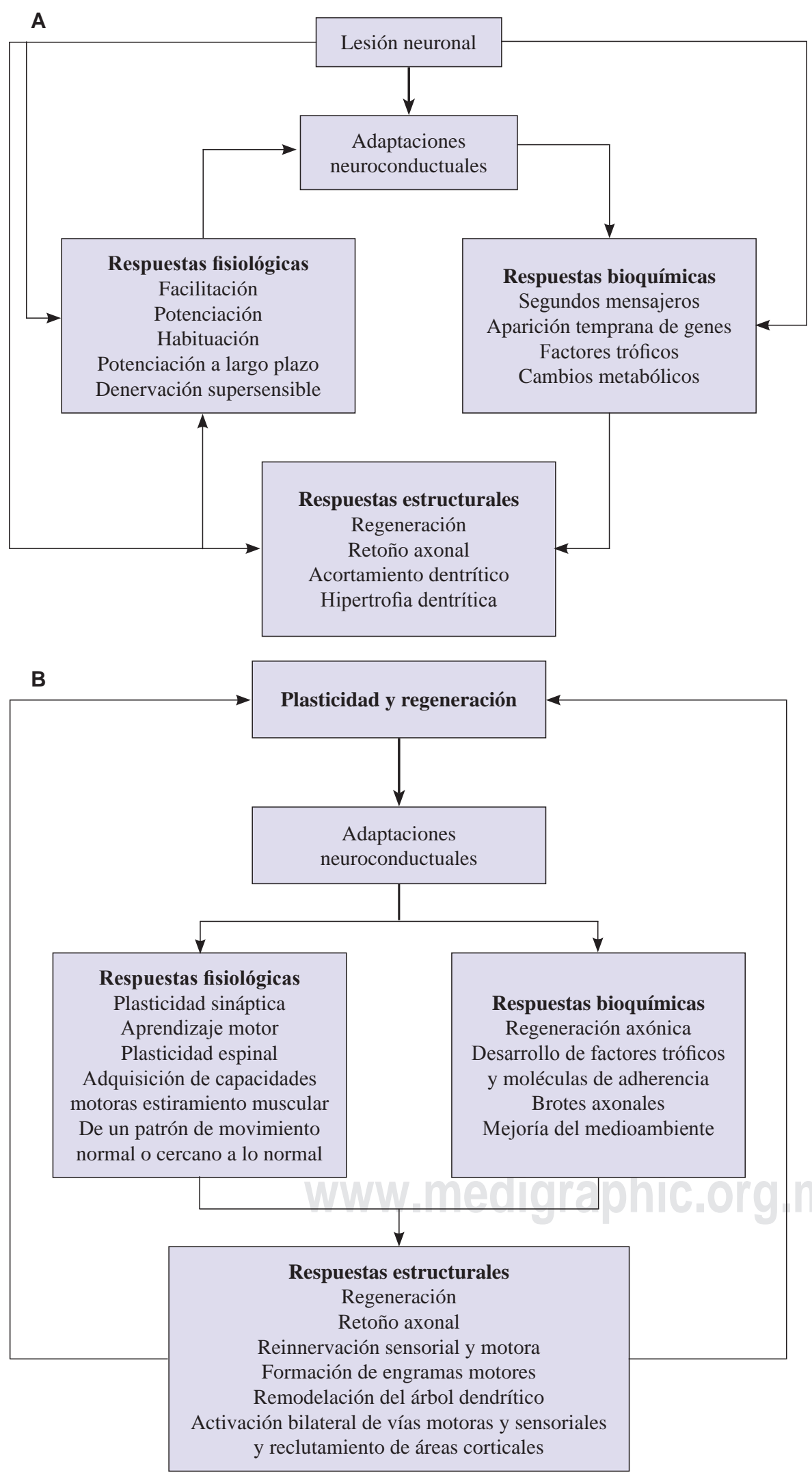

Figura 5:

A) Mecanismos de lesión nerviosa después del daño cerebral. B) Algunos mecanismos de plasticidad y regeneración cerebral. 
Asimismo, la Organización Mundial de la Salud (OMS) desde 1982 define el término neuroplasticidad como: la capacidad de las células del sistema nervioso para regenerarse anatómica y funcionalmente, después de estar sujetas a influencias patológicas ambientales o del desarrollo, incluyendo traumatismos y enfermedades. A través de este conocimiento se abre la posibilidad de ayudar al cerebro a construirse, facilitando sus propios mecanismos de plasticidad cerebral. Quizá logremos demostrar que las armas que la neurociencia ofrece hoy para fomentar la recuperación funcional de la corteza motora o somatosensorial pueden aplicarse a los mecanismos que rigen la cognición y la patología neuropsicológica. Y también sabemos el desarrollo de las funciones sensitivas como la visión, la audición y el lenguaje, cada uno con sus periodos críticos correspondientes. Si un niño no oye no desarrolla el habla, es decir, si es sordo no recibe estímulo el cerebro y será mudo -sordomudo-, al detectarlos se corregirá con implante coclear. Existen otros periodos críticos para diversas funciones durante el neurodesarrollo, por ejemplo, para el aprendizaje de las matemáticas y para el de funciones que requieren orientación espacial como la geografía y la historia, entre otras.

Recordemos que tanto la falta de estimulación como la intervención temprana y la rehabilitación son capaces de modular la actividad gabaérgica básica para iniciar los cambios neuroplásticos implicados en la recuperación funcional, lo cual permite nuevas posibilidades de estudio y acercamiento a diversas patologías y su recuperación, y quizá nos ayude a encontrar, desde la humildad del desconocimiento, la clave para entrar en el cerebro de los niños con necesidades especiales.

\section{REFERENCIAS}

1. Aguilar RF. Avances en la restauración del sistema nervioso. Editorial CRECE 2003.

2. Aguilar RF. Plasticidad cerebral y restauración neurológica. Editorial Vicoba, 1990.

3. Aguilar RF. Plasticidad neuronal parte 1. Rev Med IMSS. 2003; 41 (1): 55-64.

4. Aguilar RF. Plasticidad cerebral parte 2. Rev Med IMSS. 2003; 41 (2): 133-142.

5. Aguilar R. Avances en la restauración del sistema nervioso. Bol Med Hosp Infant Mex. 1996.

6. Fuchs $\mathrm{E}$, Flügge $\mathrm{G}$. Adult neuroplasticity: more than 40 years of research. Neural Plasticity. 2014.

7. Reznikov, Leah R, Fadel Jim R.Reagan, Lawrence P. Glutamatemediated neuroplasticity deficits in mood disorders. In: Costa e Silva JA, Macher JP, Olié JP eds. Neuroplasticity: New biochemical mechanisms. SpringerLink: Bücher. London: Springer Healthcare. p. 13. ISBN 9781908517180 . Archived from the original on 6 August 2020. Retrieved 11 July 2020.

8. Davidson RJ, McEwen BS. Social influences on neuroplasticity: stress and interventions to promote well-being. Nature Neuroscience. 2012; 15 (5): 689-695.

9. Park DC, Huang CM. Culture wires the brain. Perspectives on Psychological Science. 2010; 5 (4): 391-400.

10. Shaffer J. Neuroplasticity and clinical practice: building brain power for health. Frontiers in Psychology. 2016; 7: 1118.

11. McEwen BS. Redefining neuroendocrinology: epigenetics of brain-body communication over the life course. Frontiers in Neuroendocrinology. 2018; 49: 8-30.

12. Leuner B, Gould E. Structural plasticity and hippocampal function. Ann Rev Psychol. 2010; 61: 111-140.

13. Kusiak AN, Selzer ME. Neuroplasticity in the spinal cord. In Barnes, Michael P.; Good, David C. (eds.). Neurological Rehabilitation: Chapter 3. Neuroplasticity in the spinal cord. 3rd. China: Elsevier Inc. Chapters. ISBN 978-0-12-807792-4. Archived from the original on 13 July 2020. 2013; Retrieved 3 June 2020.

14. Livingston RB Brain mechanisms in conditioning and learning. Neurosciences Research Program Bulletin. 2017; 4: 349-354.

15. Rakic P. Neurogenesis in adult primate neocortex: an evaluation of the evidence. Nat Rev Neurosci. 2002; 3 (1): 65-71. 